\title{
290 metagenome-assembled genomes from the Mediterranean Sea: a resource for marine microbiology
}

\author{
Benjamin J Tully ${ }^{\text {Corresp., }}{ }^{1}$ ， Rohan Sachdeva ${ }^{2}$ ， Elaina D Graham ${ }^{2}$ ， John F Heidelberg ${ }^{1,2}$ \\ ${ }^{1}$ Center for Dark Energy Biosphere Investigations, University of Southern California, Los Angeles, California, USA \\ 2 Department of Biological Sciences, University of Southern California, Los Angeles, California, United States \\ Corresponding Author: Benjamin J Tully \\ Email address: tully.bj@gmail.com
}

The Tara Oceans Expedition has provided large, publicly-accessible microbial metagenomic datasets from a circumnavigation of the globe. Utilizing several size fractions from the samples originating in the Mediterranean Sea, we have used current assembly and binning techniques to reconstruct 290 putative draft metagenomeassembled bacterial and archaeal genomes, with an estimated completion of $\geq 50 \%$, and an additional 2,786 bins, with estimated completion of $0-50 \%$. We have submitted our results, including initial taxonomic and phylogenetic assignments, for the putative draft genomes to open-access repositories for the scientific community to use in ongoing research. 


\title{
290 Metagenome-assembled Genomes from the Mediterranean Sea: a resource for marine microbiology
}

\author{
Authors \\ Benjamin J. Tully ${ }^{1 \ddagger}$, Rohan Sachdeva², Elaina D. Graham² , and John F. Heidelberg ${ }^{1,2}$ \\ + - corresponding author, tully.bj@gmail.com \\ 1 - Center for Dark Energy Biosphere Investigations, University of Southern California, Los \\ Angeles, CA 90089 \\ 2 - Department of Biological Sciences, University of Southern California, Los Angeles, CA \\ 90089
}

\begin{abstract}
The Tara Oceans Expedition has provided large, publicly-accessible microbial metagenomic datasets from a circumnavigation of the globe. Utilizing several size fractions from the samples originating in the Mediterranean Sea, we have used current assembly and binning techniques to reconstruct 290 putative draft metagenome-assembled bacterial and archaeal genomes, with an estimated completion of $\geq 50 \%$, and an additional 2,786 bins, with estimated completion of 0 $50 \%$. We have submitted our results, including initial taxonomic and phylogenetic assignments, for the putative draft genomes to open-access repositories for the scientific community to use in ongoing research.
\end{abstract}

\section{Introduction}

Microorganisms are a major constituent of the biology within the world's oceans and act as important linchpins in all major global biogeochemical cycles (Falkowski, Fenchel \& DeLong, 2008). Marine microbiology is among the disciplines at the forefront in understanding how microorganisms respond to and impact local and large-scale environments. An estimated $10^{29}$ Bacteria and Archaea (Whitman, Coleman \& Wiebe, 1998) reside in the oceans and represent an immense amount of poorly constrained, and ever evolving genetic diversity.

The Tara Oceans Expedition (2003-2010) was a major endeavor to add to the body of knowledge collected during previous global ocean surveys to sample the genetic potential of microorganisms (Karsenti et al., 2011). To accomplish this goal, Tara Oceans sampled planktonic organisms (viruses to fish larvae) at two major depths, the surface ocean and the mesopelagic. The amount of data collected was expansive and included 35,000 samples from 210 ecosystems (Karsenti et al., 2011). The Tara Oceans Expedition generated and publically released 7.2 Tbp of metagenomic data from 243 ocean samples from throughout the global ocean, specifically targeting the smallest members of the ocean biosphere, the viruses, Bacteria and Archaea, and picoeukaryotes (Sunagawa et al., 2015). Initial work on these fractions produced a large protein database, totaling $>40$ million nonredundant protein sequences and identified >35,000 microbial operational taxonomic units (OTUs) (Sunagawa et al., 2015).

Leveraging the publically available metagenomic sequences from the "girus" (giant virus; 0.22-1.6 $\mu \mathrm{m})$, "bacteria" $(0.22-1.6 \mu \mathrm{m})$, and "protist" $(0.8-5 \mu \mathrm{m})$ size fractions, we have performed a new joint assembly of these samples using current sequence assemblers (Megahit (Li et al., 2016)) and methods (combining assemblies from multiple sites using Minimus2 (Treangen et al., 2011)). These metagenomic assemblies were binned using BinSanity (Graham, Heidelberg \& Tully, 2017) in to 290 draft microbial genomes with an estimated completeness 
47 ranging from 50-100\%. Environmentally derived genomes are imperative for a number of

48 downstream applications, including comparative genomics, metatranscriptomics, and

49

50

51

52

53

54

55

56

57

58

59

60

61

62

63

64

65

66

67

68

69

70

71

72

73

74

75

76

77

78

79

80

81

82

83

84

85

86

87

88

89

90

91

92

metaproteomics. This series of genomic data can allow for the recruitment of environmental "omic" data and provide linkages between functions and phylogenies. This method was initially performed on the seven sites from the Mediterranean Sea containing microbial metagenomic samples (TARA007, -009, -018, -023, -025 and -030), but will continue through the various Longhurst provinces (Longhurst et al., 1995) sampled during the Tara Oceans project (Figure 1). All of the assembly data is publically available, including the initial Megahit assemblies for each site from the various size fractions and depths along with the recovered putative (minimal quality control) genomes.

\section{Materials and Methods}

A generalized version of the following workflow is presented in Figure 2.

Sequence Retrieval and Assembly

All sequences for the reverse and forward reads from each sampled site and depth within the Mediterranean Sea were accessed from European Molecular Biology Laboratory-European Bioinformatics Institute (EMBL-EBI) utilizing their FTP service (Table 1). Paired-end reads from different filter sizes from each site and depth (e.g., TARA0007, girus filter fraction, sampled at the deep chlorophyll maximum) were assembled using Megahit (Li et al., 2016) (v1.0.3; parameters: --preset, meta-sensitive). To keep consistent with TARA sample nomenclature, "bacteria" or "BACT" will be used to encompass the size fraction 0.22-1.6 $\mu \mathrm{m}$. Megahit assemblies $\geq 2 \mathrm{~kb}$ in length from all samples were pooled and combined based on $\geq 99 \%$ semi-global identity using CD-HIT-EST (Fu et al., 2012) (v4.6; -c 0.99) in order to reduce the number of redundant contigs for the downstream assembly step. The reduced set of contiguous DNA fragments (contigs) was then cross-assembled based on a minimum of 100bp overlaps with 95\% nucleotide identity using Minimus2 (Treangen et al., 2011) (AMOS v3.1.0; parameters: -D OVERLAP=100 MINID=95). This assembly method is available on Protocols.io at https://dx.doi.org/10.17504/protocols.io.hfqb3mw.

\section{Metagenome-assembled Genomes}

Due to memory limitations during the binning step, contigs $\geq 7.5 \mathrm{~kb}$ in length generated during the two-step assembly process (includes Minimus 2 contigs and unincorporated Megahit contigs) were used to recruit sequence reads from each of the Tara samples using Bowtie2 (Langmead \& Salzberg, 2012) (v4.1.2; default parameters). Read counts for each contig were determined using featureCounts (Liao, Smyth \& Shi, 2014) (v1.5.0; default parameters). Coverage was determined for all contigs by dividing the number of recruited reads by the length of the contig (reads/bp). Due to the low coverage nature of the samples, in order to effectively delineate between contig coverage patterns, the coverage values were transformed by multiplying by five (determined through manual tuning). Transformed coverage values were then utilized to cluster contigs in to bins utilizing BinSanity (Graham, Heidelberg \& Tully, 2017) based on a preference value of -3 to be run for a maximum of 4,000 iterations with completion if convergence is achieved for 400 consecutive iterations and a damping value of 0.9 (parameters: $\mathrm{p}-3,-\mathrm{m} 4000,-\mathrm{v} 400,-\mathrm{d} 0.9$ ). Bins were assessed for the presence of putative microbial genomes using the lineage workflow in CheckM (Parks et al., 2015) (v1.0.3; parameters: lineage_wf). 
93 Bins were split in to three categories: (1) putative draft genomes $(\geq 50 \%$ complete and $\leq 10 \%$

94 cumulative redundancy [\% contamination $-(\%$ contamination $\times \%$ strain heterogeneity $\div 100)])$;

95 (2) draft genomes with high contamination ( $\geq 50 \%$ complete and $\geq 10 \%$ cumulative redundancy);

96 and (3) low completion bins ( $<50 \%$ complete).

97

The high contamination bins containing approximately two genomes, three genomes, or $\geq 4$ genomes used BinSanity (Binsanity; -m 2000, -v 200, -d 0.9) with variable preference values $(-p)$ of $-1000,-500$, and -100 , respectively. The resulting bins were added to one of the three categories: putative draft genomes, high contamination bins, and low completion bins. The high contamination bins were processed for a third time with the Binsanity-refine utilizing a preference of $-100(-p-100)$. These bins were given final assignments as either putative draft genomes or low completion bins.

Any contigs not assigned to putative draft genomes were assessed using BinSanity using raw coverage values. Two additional rounds of refinement were performed with the first round of refinement using preference values based on the estimated number of contaminating genomes (as above) and the second round using a set preference of $-10(-\mathrm{p}-10)$. Following this binning phase, contigs were assigned to draft genome bins (e.g., Tara Mediterranean genome 1, referred to as TMED1, etc.), low completion bins with at least five contigs (0-50\% complete; TMEDlc1, etc. lc, low completion), or were not placed in a bin (Supplemental Information S1 \& S2).

\section{Taxonomic and Phylogenetic Assignment of Draft Genomes}

The bins representing the draft genomes were assessed for taxonomy and phylogeny using multiple methods to provide a quick reference for selecting genomes of interest. Taxonomy was assigned using the putative placement provided via CheckM during the pplacer (Matsen, Kodner \& Armbrust, 2010) step of the analysis to the lowest taxonomic placement (parameters: tree_qa -o 2). This step was also performed for all low completion bins.

Two separate attempts were made to assign the draft genomes a phylogenetic assignment.

119

120

121 Draft genomes were searched for the presence of the full-length 16S rRNA gene sequence using RNAmmer (Lagesen et al., 2007) (v1.2; parameters: -S bac -m ssu). All full-length sequences were aligned to the SILVA SSU reference database (Ref123) using the SINA web portal aligner (Pruesse, Peplies \& Glöckner, 2012) (https://www.arb-silva.de/aligner/). These alignments were loaded in to ARB (Ludwig et al., 2004) (v6.0.3), manually assessed, and added to the nonredundant 16S rRNA gene database (SSURef123 NR99) using ARB Parsimony (Quick) tool (parameters: default). A selection of the nearest neighbors to the Tara genome sequences were selected and used to construct a 16S rRNA phylogenetic tree. Genome-identified 16S rRNA sequences and SILVA reference sequences were aligned using the SINA web portal aligner (Supplemental Information S4). An approximately-maximum-likelihood tree with ShimodairaHasegawa local support values (Shimodaira \& Hasegawa, 1999) was constructed using FastTree (Price, Dehal \& Arkin, 2010) using the generalized time reversible and discrete gamma models (Yang) (v2.1.3; parameters: -nt -gtr -gamma; Figure 3; Supplemental Information S5).

Draft genomes were assessed for the presence of the 16 ribosomal markers genes used in Hug, et al. (2016). Putative CDSs were determined using Prodigal (v2.6.3; parameters: -m -p meta) and were searched using HMMs for each marker using HMMER (Finn, Clements \& Eddy, $2011)$ with matches based on an e-value cutoff of $1 \times 10^{-3}$ (v3.1b2; parameters: hmmsearch -E 1E10 ). If a genome had multiple copies of any single marker gene, neither was considered, and only genomes with $\geq 8$ markers were used to construct a phylogenetic tree. Markers identified from the draft genomes were combined with markers from 6,080 reference genomes accessed from 
139 NCBI GenBank (Supplemental Information S6) that represent the major bacterial phylogenetic 140 groups. Each marker gene was aligned using MUSCLE (Edgar, 2004) (parameters: -maxiters 8) 141 and automatically trimmed using trimAL (Capella-Gutiérrez, Silla-Martínez \& Gabaldón, 2009) 142 (v1.2rev59; parameters: -automated1). Automated trimming results were manually curated in 143 Geneious (Kearse et al., 2012) (Supplemental Information S7). Final alignments were

144 concatenated and used to construct an approximately-maximum-likelihood tree using the LG (Le 145 \& Gascuel, 2008) and Gamma models with Shimodaira-Hasegawa local support values with 146 FastTree (v2.1.10; parameters: -lg -gamma; Figure 4; Supplemental Information S8). A separate tree was constructed using the same concatenated alignments and tree-building parameters for the 210 draft genomes, without the reference genomes (Figure 4).

\section{Relative Abundance of Draft Genomes}

To set-up a baseline that could approximate the "microbial" community (Bacteria, Archaea and viruses) present in the various Tara metagenomes, which included filter sizes specifically targeting both protists and giruses, reads were recruited against all contigs generated from the Minimus2 and Megahit assemblies $\geq 2 \mathrm{~kb}$ using Bowtie2 (default parameters). The contigs $<2 \mathrm{~kb}$ in length likely constitute low abundance bacteria and archaea, bacteria and archaea with high degrees of repeats resulting in poor assembly, fragmented picoeukaryotic genomes, and problematic read sequences (low quality, sequencing artefacts, etc.) and were not included in further analysis. All relative abundance measures are relative to the number of reads recruited to the assemblies $\geq 2 \mathrm{~kb}$. Read counts were determined using featureCounts (as above). Lengthnormalized relative abundance values were determined for each draft genome for each sample:

$$
\frac{\frac{\text { Reads }}{b p} \text { per genome }}{\sum \frac{\text { Reads }}{b p} \text { all genomes }} \times \frac{\sum \text { Recruited reads to genomes }}{\sum \text { Recruited reads to all contigs }(\geq 2 \mathrm{~kb})} \times 100
$$

\section{Data Availability}

This project has been deposited at DDBJ/ENA/GenBank under the BioProject accession no. PRJNA385857 and drafts of genomes are available with accession no. NHBG00000000NHMJ00000000. Additional files have been provided and are available through FigShare (https://dx.doi.org/10.6084/m9.figshare.3545330), such as: all contigs from Minimus2 + Megahit output used for binning and community assessment; contig read counts per sample; the putative genome contigs and Prodigal-predicted nucleotide and protein putative CDS FASTA files; the ribosomal marker HMM profiles; reference genome markers; draft genome markers; low completion bins, and contigs without a bin. All contigs generated using Megahit from each sample are available through iMicrobe (http://data.imicrobe.us/project/view/261). TARA Oceans has deposited the reads in the NCBI Sequence Read Archive (SRA) with accessions ERS488346, ERS488330, ERS477998, ERS477979, ERS488509, ERS488486, ERS478040, ERS477953, ERS477931, ERS488147, ERS488119, and ERS478017. 
180

181

182

183

184

185

186

187

188

189

190

191

192

193

194

195

196

197

198

199

200

201

202

203

204

205

206

207

208

209

210

211

212

213

214

215

216

217

218

219

220

221

222

223

224

The initial Megahit assembly was performed on the publicly available reads for Tara stations $007,009,018,023,025,030$. Starting with 147-744 million reads per sample, the Megahit assembly process generated 1.2-4.6 million contigs with a mean $N_{50}$ and longest contig of $785 \mathrm{bp}$ and $537 \mathrm{~kb}$, respectively (Table 1). In general, the contigs generated from the Tara samples targeting the protist size fraction $(0.8-5 \mu \mathrm{m})$ had a shorter $N_{50}$ value than the bacteria size fractions (mean: $554 \mathrm{bp}$ vs $892 \mathrm{bp}$, respectively). Contigs from the Megahit assembly process were pooled and separated by length. Of the 42.6 million contigs generated during the first assembly, 1.5 million were $\geq 2 \mathrm{~kb}$ in length (Table 2 ). Several attempts were made to assemble the shorter contigs, but publicly available overlap-consensus assemblers (Newbler [454 Life Sciences], cap3 (Huang \& Madan, 1999), and MIRA (Chevreux et al., 2004)) failed on multiple attempts. Processing the $\geq 2 \mathrm{~kb}$ contigs from all of the samples through CD-HIT-EST reduced the total to 1.1 million contigs. This group of contigs was subjected to the secondary assembly through Minimus2, generating 158,414 new contigs (all $\geq 2 \mathrm{~kb}$ ). The secondary contigs were combined with the Megahit contigs that were not assembled by Minimus2. This provided a contig dataset consisting of 660,937 contigs, all $\geq 2 \mathrm{~kb}$ in length (Table 2 ; further referred to as data-rich-contigs).

\section{Binning}

The set of data-rich-contigs was used to recruit the metagenomic reads from each sample using Bowtie2. The data-rich-contigs recruited $15-81 \%$ of the reads depending on the sample. In general, the protist size fraction recruited substantially fewer reads than the girus and bacteria size fractions (mean: $19.8 \%$ vs $75.0 \%$, respectively) (Table 1). For the protist size fraction, the "missing" data for these recruitments likely results from the poor assembly of more complex and larger eukaryotic genomes. The fraction of the reads that do not recruit in the girus and bacterial size fraction samples could be accounted for by the large number of low quality assemblies (200$500 \mathrm{bp}$ ) and reads that could not be assembled due to low abundance or high complexity (Table 2).

Unsupervised binning was performed using both transformed and raw coverage values for a subset of 95,506 contigs from the data-rich-contigs that were $\geq 7.5 \mathrm{~kb}$ (referred to further as binned-contigs). Binning using the transformed coverage data generated 237 putative draft genomes containing 15,032 contigs (Supplemental Information S1). Contigs not in putative genomes were re-binned based on raw coverage values, generating 53 additional putative draft genomes encompassing 3,348 contigs. In total, 290 putative draft genomes were generated with $50-100 \%$ completion (mean: 69\%) with a mean length and number of putative CDS of $1.7 \mathrm{Mbp}$ and 1,699, respectively (Supplemental Information S1). In analyzing the quality of generated draft genomes, 31 of the genomes had a contamination value $>10 \%$, but had a cumulative redundancy value $<10 \%$, while an additional 12 genomes had a cumulative redundancy value $>10 \%$ (genomes highlighted in Supplemental Information S1). For instances where the predicted strain heterogeneity is high and sharply reduces the calculated cumulative redundancy value (e.g., TMED1), downstream analysis of these genomes may offer opportunities to examine within strain variation, if the gene content varies between contigs, or identify duplicate contigs that can be removed to rectify contamination issues. For instances where cumulative redundancy values remain high (e.g., TMED20 or TMED106), downstream analysis utilizing composition signatures, for example within Anvi'o (Eren et al., 2015), should be able to identify problematic contigs and reduce the overall contamination reported for the genome. 
251

252

253

254

255

256

257

258

259

260

261

262

263

264

265

266

267

268

269

270

All other contigs were grouped in to bins with at least five contigs, but with estimated completion of $0-50 \%$ (2,786 low completion bins; 74,358 contigs; Supplemental Information S2) or did not bin (2,732 contigs). Nearly a quarter of the 2,786 low completion bins $(24.7 \%)$ have an estimated completion of $0 \%$. These bins may be good candidates for exploring small doublestranded viral genomes or episomal genetic elements.

\section{Taxonomy, Phylogeny, \& Potential Organisms of Interest}

The 290 putative draft genomes had a taxonomy assigned to it via CheckM during the pplacer step. All of the genomes, except for 20, had an assignment to at least the Phylum level, and $83 \%$ of the genomes had an assignment to at least the Class level (Supplemental Information S1).

Phylogenetic information was determined for as many genomes as possible. Genomes were assessed for the presence of full-length 16S rRNA genes. In total, 37 16S rRNA genes were detected in 35 genomes. 16S rRNA genes can prove to be problematic during the assembly steps due the high level of conservation that can break contigs (Miller, Koren \& Sutton, 2010) (Figure $3)$. The conserved regions of the $16 \mathrm{~S}$ rRNA, depending on the situation, can over- or underrecruit reads, resulting in coverage variations that can misplace contigs in to the incorrect genome. As such, most of the 16S rRNA phylogenetic placements support the taxonomic assignments, while eight of the assignments were contradictory in nature (denoted in Supplemental Information S1). An example of this nonconformity of 16S rRNA assignment would be TMED32. A Bacteroidetes, TMED32 is assigned to the Order Cytophagales via CheckM and the ribosomal marker tree and contains three 16S rRNA sequences. One of the 16S rRNA sequences is conformational, with placement in the Family Flammeovirgaceae, while the remaining two 16S rRNA are assigned to the Mitochondria. For future research purposes, contigs with contradictory $16 \mathrm{~S}$ rRNA or incongruent phylogenetic/taxonomic signatures, should be removed. Downstream analysis should allow for the determination of the most parsimonious result in any draft genome with contradictory phylogenetic assignments.

Beyond the 16S rRNA gene, genomes were searched for 16 conserved, syntenic ribosomal markers. Sufficient markers $(\geq 8)$ were identified in 210 of the genomes $(72 \%)$ and placed on a tree with 6,080 reference sequences and used to assign a putative phylogeny (Supplemental Information S1). Phylogenies were then assigned to the lowest taxonomic level that could be confidently determined (Figure 4). These putative results reveal a number of genomes were generated that represent multiple clades for which environmental genomic information remains limited, including: Planctomycetes, Verrucomicrobia, Cyanobacteria, and uncultured groups within the Alpha- and Gammaproteobacteria.

\section{Relative Abundance}

A length-normalized relative abundance value was determined for each genome in each sample based on the number of reads recruited to the data-rich-contigs. The relative abundance for the individual genomes was determined based on this portion of the dataset (Supplemental Information S3). In general, the genomes had low relative abundance (maximum relative abundance $=1.9 \%$ for TMED155 a putative Cyanobacteria at site TARA023 from the protistan size fraction sampled at the surface; Supplemental Information S1). The draft genomes accounted for $1.57-25.16 \%$ of the approximate microbial community as determined by the datarich-contigs (mean $=13.69 \%$ ), with the ten most abundant genomes in a sample representing $0.61-10.31 \%$ (Table 1$)$. 
271

272

273

274

275

276

277

278

279

280

281

282

283

284

285

286

287

288

289

290

291

292

293

294

295

296

297

298

299

300

301

302

303

304

305

306

307

308

309

310

311

312

313

314

315

316

\section{Concluding Statement}

The goal of this project was to provided preliminary putative genomes from the Tara Oceans microbial metagenomic datasets. The 290 putative draft genomes and 2,786 low completion bins were created using the 20 samples and six stations from the Mediterranean Sea.

Initial assessment of the phylogeny of these metagenomic-assembled genomes based on concatenated ribosomal markers indicates several new genomes from environmentally relevant organisms, including, approximately 10 new Cyanobacteria genomes within the genera Prochlorococcus and Synechococcus and 22 new SAR11 genomes. Additionally, there are putative genomes from the marine Euryarchaeota $(\mathrm{n}=13)$, Verrucomicrobia $(\mathrm{n}=15)$, and Planctomycetes $(\mathrm{n}=7)$. Additionally, the low completion bins may house distinct viral genomes. Of particular interest may be the 40 bins with $0 \%$ completion (based on single-copy marker genes), but that contain $>500 \mathrm{~kb}$ of genetic material (including 3 bins with $>1 \mathrm{Mb}$ ). These large bins lacking markers may be good candidates for research in to the marine "giant viruses" and episomal DNA sources (plasmids, etc.).

It should be noted, researchers using this dataset should be aware that all of the genomes generated from these samples should be used as a resource with some skepticism towards the results being an absolute. Like all results for metagenome-assembled genomes, these genomes represent a best-guess approximation of a taxon from the environment (Sharon \& Banfield, 2013). Researchers are encouraged to confirm all claims through various genomic analyses and accuracy may require the removal of conflicting sequences.

\section{Acknowledgements}

We are indebted to the Tara Oceans project and team for their commitment to openaccess data that allows data aficionados to indulge in the data and attempt to add to the body of science contained within. This is C-DEBI Contribution XXX.

\section{References}

Capella-Gutiérrez S, Silla-Martínez JM, Gabaldón T 2009. trimAl: a tool for automated alignment trimming in large-scale phylogenetic analyses. Bioinformatics 25:1972-1973.

Chevreux B, Pfisterer T, Drescher B, Driesel AJ, Müller WEG, Wetter T, Suhai S 2004. Using the miraEST assembler for reliable and automated mRNA transcript assembly and SNP detection in sequenced ESTs. Genome Research 14:1147-1159.

Edgar RC 2004. MUSCLE: multiple sequence alignment with high accuracy and high throughput. Nucleic Acids Research 32:1792-1797.

Eren AM, Esen ÖC, Quince C, Vineis JH, Morrison HG, Sogin ML, Delmont TO 2015. Anvi“o: an advanced analysis and visualization platform for "omics data. PeerJ 3:e1319.

Falkowski PG, Fenchel T, DeLong EF 2008. The Microbial Engines That Drive Earth's Biogeochemical Cycles. Science 320:1034-1039.

Finn RD, Clements J, Eddy SR 2011. HMMER web server: interactive sequence similarity searching. Nucleic Acids Research 39:W29-W37.

Fu L, Niu B, Zhu Z, Wu S, Li W 2012. CD-HIT: accelerated for clustering the next-generation sequencing data. Bioinformatics 28:3150-3152.

Graham ED, Heidelberg JF, Tully BJ 2017. BinSanity: unsupervised clustering of environmental microbial assemblies using coverage and affinity propagation. PeerJ 5:e3035-19. 
317

318

319

320

321

322

323

324

325

326

327

328

329

330

331

332

333

334

335

336

337

338

339

340

341

342

343

344

345

346

347

348

349

350

351

352

353

354

355

356

357

358

359

360

361

362

Huang X, Madan A 1999. CAP3: A DNA sequence assembly program. Genome Research 9:868-877.

Hug LA, Baker BJ, Anantharaman K, Brown CT, Probst AJ, Castelle CJ, Butterfield CN, Hernsdorf AW, Amano Y, Ise K, Suzuki Y, Dudek N, Relman DA, Finstad KM, Amundson R, Thomas BC, Banfield JF 2016. A new view of the tree of life. Nature Microbiology:1-6.

Karsenti E, Acinas SG, Bork P, Bowler C, de Vargas C, Raes J, Sullivan M, Arendt D, Benzoni F, Claverie J-M, Follows M, Gorsky G, Hingamp P, Iudicone D, Jaillon O, Kandels-Lewis S, Krzic U, Not F, Ogata H, Pesant S, Reynaud EG, Sardet C, Sieracki ME, Speich S, Velayoudon D, Weissenbach J, Wincker P, the Tara Oceans Consortium 2011. A Holistic Approach to Marine Eco-Systems Biology. PLoS Biology 9:e1001177-5.

Kearse M, Moir R, Wilson A, Stones-Havas S, Cheung M, Sturrock S, Buxton S, Cooper A, Markowitz S, Duran C, Thierer T, Ashton B, Mentjies P, Drummond A 2012. Geneious Basic: an integrated and extendable desktop software platform for the organization and analysis of sequence data. Bioinformatics 28:1647-1649.

Lagesen K, Hallin P, Rødland EA, Staerfeldt H-H, Rognes T, Ussery DW 2007. RNAmmer: consistent and rapid annotation of ribosomal RNA genes. Nucleic Acids Research 35:31003108 .

Langmead B, Salzberg SL 2012. Fast gapped-read alignment with Bowtie 2. Nature Methods 9:357-359.

Le SQ, Gascuel O 2008. An Improved General Amino Acid Replacement Matrix. Molecular Biology and Evolution 25:1307-1320.

Li D, Luo R, Liu C-M, Leung C-M, Ting H-F, Sadakane K, Yamashita H, Lam T-W 2016. MEGAHIT v1.0: A fast and scalable metagenome assembler driven by advanced methodologies and community practices. Methods 102:3-11.

Liao Y, Smyth GK, Shi W 2014. featureCounts: an efficient general purpose program for assigning sequence reads to genomic features. Bioinformatics 30:923-930.

Longhurst A, Sathyendranath S, Platt T, Caverhill C 1995. An Estimate of Global Primary Production in the Ocean From Satellite Radiometer Data. Journal of Plankton Research 17:1245-1271.

Ludwig W, Strunk O, Westram R, Richter L, Meier H, Yadhukumar, Buchner A, Lai T, Steppi S, Jobb G, Förster W, Brettske I, Gerber S, Ginhart AW, Gross O, Grumann S, Hermann S, Jost R, König A, Liss T, Lüssmann R, May M, Nonhoff B, Reichel B, Strehlow R, Stamatakis A, Stuckmann N, Vilbig A, Lenke M, Ludwig T, Bode A, Schleifer K-H 2004. ARB: a software environment for sequence data. Nucleic Acids Research 32:1363-1371.

Matsen FA, Kodner RB, Armbrust EV 2010. pplacer: linear time maximum-likelihood and Bayesian phylogenetic placement of sequences onto a fixed reference tree. $B M C$ bioinformatics 11:538.

Miller JR, Koren S, Sutton G 2010. Assembly algorithms for next-generation sequencing data. Genomics 95:315-327.

Parks DH, Imelfort M, Skennerton CT, Hugenholtz P, Tyson GW 2015. CheckM: assessing the quality of microbial genomes recovered from isolates, single cells, and metagenomes. Genome Research 25:1043-1055.

Price MN, Dehal PS, Arkin AP 2010. FastTree 2--approximately maximum-likelihood trees for large alignments. PLoS ONE 5:e9490.

Pruesse E, Peplies J, Glöckner FO 2012. SINA: accurate high-throughput multiple sequence alignment of ribosomal RNA genes. Bioinformatics 28:1823-1829.

PeerJ reviewing PDF | (2017:05:17890:1:1:CHECK 13 Jun 2017) 
363 364

365

366

367

368

369

370

371

372

373

374

375

376

377

378

379

380

381

382

383

Sharon I, Banfield JF 2013. Microbiology. Genomes from metagenomics. Science 342:10571058.

Shimodaira H, Hasegawa M 1999. Multiple comparisons of log-likelihoods with applications to phylogenetic inference. Molecular Biology and Evolution 16:1114-1116.

Sunagawa S, Coelho LP, Chaffron S, Kultima JR, Labadie K, Salazar G, Djahanschiri B, Zeller G, Mende DR, Alberti A, Cornejo-Castillo FM, Costea PI, Cruaud C, d'Ovidio F, Engelen S, Ferrera I, Gasol JM, Guidi L, Hildebrand F, Kokoszka F, Lepoivre C, Lima-Mendez G, Poulain J, Poulos BT, Royo-Llonch M, Sarmento H, Vieira-Silva S, Dimier C, Picheral M, Searson S, Kandels-Lewis S, Tara Oceans Coordinators, Bowler C, de Vargas C, Gorsky G, Grimsley N, Hingamp P, Iudicone D, Jaillon O, Not F, Ogata H, Pesant S, Speich S, Stemmann L, Sullivan MB, Weissenbach J, Wincker P, Karsenti E, Raes J, Acinas SG, Bork P 2015. Ocean plankton. Structure and function of the global ocean microbiome. Science 348:1261359-1261359.

Treangen TJ, Sommer DD, Angly FE, Koren S, Pop M 2011. Next generation sequence assembly with AMOS. Current protocols in bioinformatics / editoral board, Andreas D. Baxevanis ... [et al.] Chapter 11:Unit 11.8.

Whitman WB, Coleman DC, Wiebe WJ 1998. Prokaryotes: The unseen majority. Proceedings of the National Academy of Sciences of the United States of America 95:6578-6583.

Yang Z Maximum likelihood phylogenetic estimation from DNA sequences with variable rates over sites: Approximate methods. Journal of Molecular Evolution 39:306-314. 
Figure 1

Figure 1

Map illustrating the locations and size fractions sampled for the Tara Oceans Mediterranean Sea datasets. Girus, 'giant virus' size fraction (0.22-1.6 $\mu \mathrm{m})$. Bact, 'bacteria' size fraction (0.22-1.6 $\mu \mathrm{m})$. Prot, 'protist' size fraction $(0.8-5.0 \mu \mathrm{m})$. The map in Figure 1 was modified under a CC BY-SA 3.0 license from 'Blank Map of South Europe and North Africa' by historicair (https://upload.wikimedia.org/wikipedia/commons/d/db/Blank_map_of_South_Europe_and_Nor th_Africa.svg).

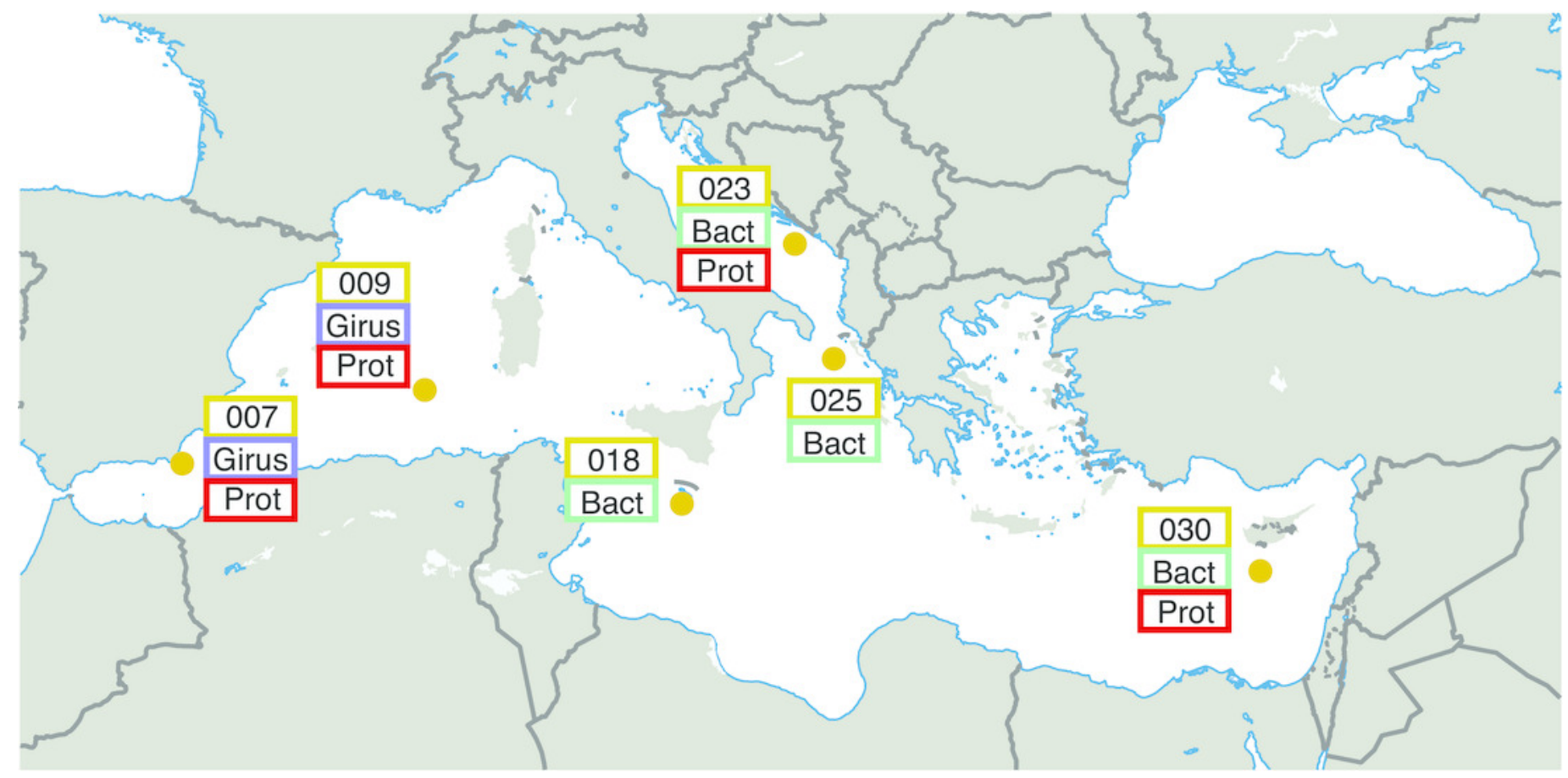


Figure 2

Figure 2

Workflow used to process Tara Oceans Mediterranean Sea metagenomic datasets.

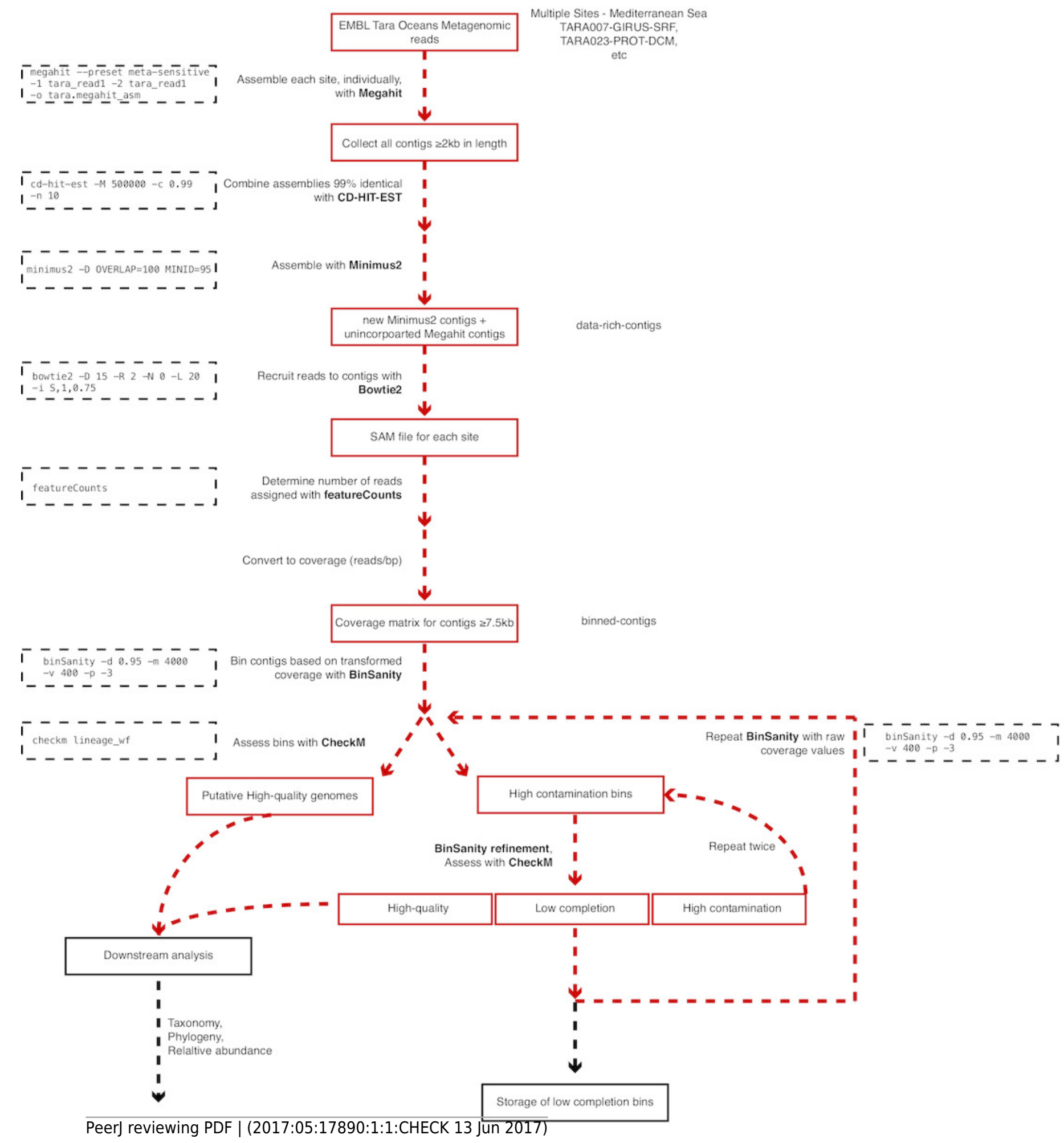




\section{Figure 3}

Figure 3

FastTree approximate maximum-likelihood phylogenetic tree constructed with 37 and 406 16S rRNA genes from putative draft genomes and references, respectively. Sequence alignment is available in Supplemental Information S4. Phylogenetic tree with ShimodairaHasegawa local support values as available in Newick format in Supplemental Information S5. 


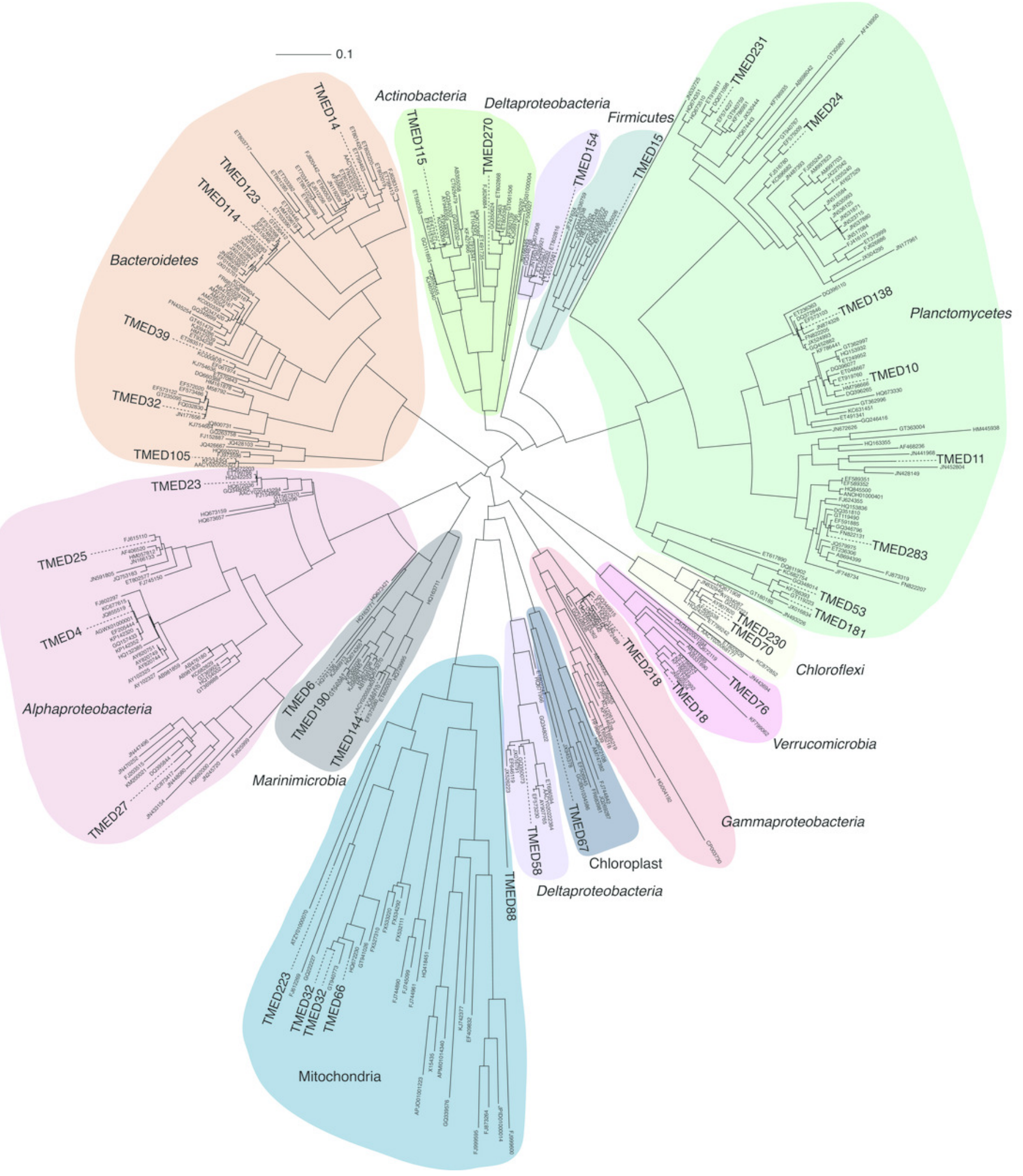




\section{Figure 4}

Figure 4

Cladogram of a FastTree approximate maximum-likelihood phylogenetic tree constructed using 16 syntenic, single-copy marker genes for 210 draft genomes. Support for internal nodes were determined based on the Shimodaira-Hasegawa test (white $\geq 0.500$, gray $\geq 0.750$, black $\geq 0.950$ ). Sequence alignment for the draft genomes and the reference genomes used for phylogenetic assignment is available in Supplemental Information S7. Phylogenetic tree of the draft genomes and the reference genomes used for phylogenetic assignment is available in Newick format in Supplemental Information S8.

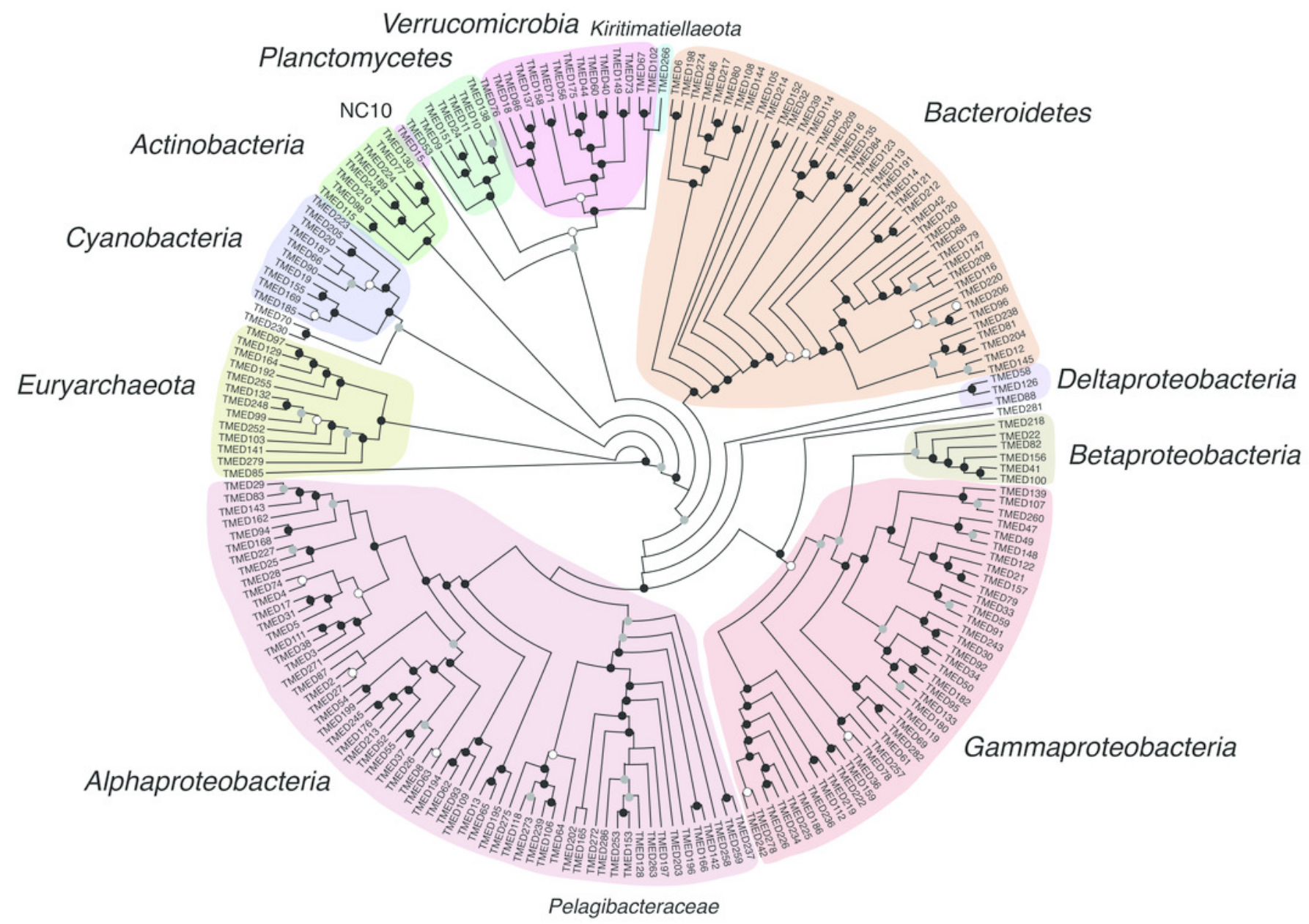




\section{Table 1 (on next page)}

Table 1

Statistics for Megahit contigs, recruitment to data-rich-contigs, and relative abundance of draft genome results for each sample. 


\begin{tabular}{|c|c|c|c|c|c|c|c|c|c|}
\hline $\begin{array}{l}\text { TARA } \\
\text { Sample } \\
\text { Site }\end{array}$ & $\begin{array}{l}\text { Size } \\
\text { Fraction } \\
\text { (Girus, } \\
\text { Bacteria, } \\
\text { or } \\
\text { Protist) }\end{array}$ & $\begin{array}{l}\text { Depth } \\
\text { (Surfac } \\
\text { e or } \\
\text { DCM }^{*} \text { ) }\end{array}$ & No. of reads & $\begin{array}{l}\text { No. of } \\
\text { initial } \\
\text { Megahit } \\
\text { assembly }\end{array}$ & $\begin{array}{l}\text { N50 (bp; } \\
\text { initial } \\
\text { Megahit } \\
\text { assembly) }\end{array}$ & $\begin{array}{l}\text { Longest } \\
\text { initial } \\
\text { Megahit } \\
\text { assembly } \\
\text { (bp) }\end{array}$ & $\begin{array}{l}\text { Recruitment } \\
\text { (\% data- } \\
\text { rich- } \\
\text { contigs })\end{array}$ & $\begin{array}{l}\text { Relative } \\
\text { abundance }^{o} \\
\text { of draft } \\
\text { genomes } \\
(\%)\end{array}$ & $\begin{array}{l}\text { Relative } \\
\text { abundance }^{\circ} \\
\text { of ten most } \\
\text { abundant } \\
\text { genomes } \\
(\%)\end{array}$ \\
\hline TARA007 & Girus & DCM & $178,519,830$ & $1,318,470$ & 828 & 220,754 & 72.84 & 14.64 & 6.35 \\
\hline TARA007 & Girus & Surface & $221,166,612$ & $1,308,847$ & 861 & 211,946 & 81.74 & 14.83 & 6.12 \\
\hline TARA007 & Protist & $\mathrm{DCM}$ & $744,458,992$ & $4,667,618$ & 654 & 188,635 & 19.45 & 8.60 & 3.18 \\
\hline TARA007 & Protist & Surface & $265,432,098$ & $2,590,120$ & 564 & 18,444 & 25.58 & 1.57 & 0.61 \\
\hline TARA009 & Girus & $\mathrm{DCM}$ & $416,553,274$ & $2,796,841$ & 831 & $1,643,839$ & 69.48 & 14.16 & 6.32 \\
\hline TARA009 & Girus & Surface & $489,617,426$ & $1,787,467$ & 929 & $1,142,851$ & 68.85 & 12.29 & 4.76 \\
\hline TARA009 & Protist & DCM & $329,036,110$ & $1,938,636$ & 613 & 95,724 & 22.07 & 13.35 & 4.20 \\
\hline TARA009 & Protist & Surface & $370,813,078$ & $1,700,350$ & 588 & 292,050 & 22.53 & 15.97 & 6.17 \\
\hline TARA018 & Bacteria & $\mathrm{DCM}$ & $408,021,182$ & $2,520,645$ & 840 & $1,573,060$ & 76.22 & 11.49 & 3.18 \\
\hline TARA018 & Bacteria & Surface & $414,976,308$ & $2,604,031$ & 816 & $2,086,508$ & 75.80 & 11.03 & 3.02 \\
\hline TARA023 & Bacteria & DCM & $147,400,552$ & $1,273,576$ & 830 & 213,456 & 76.08 & 13.29 & 4.09 \\
\hline TARA023 & Bacteria & Surface & $149,566,010$ & $1,237,617$ & 825 & 134,179 & 75.98 & 13.82 & 4.01 \\
\hline TARA023 & Protist & DCM & $508,610,652$ & $2,707,801$ & 734 & 336,689 & 28.23 & 25.07 & 7.83 \\
\hline TARA023 & Protist & Surface & $397,044,232$ & $2,246,571$ & 593 & 397,140 & 23.00 & 25.16 & 10.31 \\
\hline TARA025 & Bacteria & $\mathrm{DCM}$ & $386,627,816$ & $2,516,865$ & 806 & 388,546 & 69.77 & 14.55 & 5.35 \\
\hline TARA025 & Bacteria & Surface & $457,560,422$ & $2,326,838$ & 857 & 330,773 & 75.57 & 10.99 & 3.18 \\
\hline TARA030 & Bacteria & DCM & $346,837,034$ & $1,968,945$ & 1097 & 508,775 & 80.16 & 10.31 & 2.57 \\
\hline TARA030 & Bacteria & Surface & $478,785,582$ & $1,639,697$ & 1194 & 204,976 & 77.70 & 7.26 & 2.64 \\
\hline TARA030 & Protist & $\mathrm{DCM}$ & $426,896,616$ & $1,620,343$ & 616 & 478,892 & 15.12 & 17.83 & 5.13 \\
\hline TARA030 & Protist & Surface & $430,029,974$ & $1,838,588$ & 628 & 287,782 & 22.36 & 17.60 & 6.73 \\
\hline
\end{tabular}

1 *DCM - deep chlorophyll maximum 
$2{ }^{\mathrm{a} N 50}$ - length of DNA sequence above which $50 \%$ of the total is contained

3 'olative abundance - determined using the reads recruited data-rich-contigs 


\section{Table 2 (on next page)}

Table 2

Assembly statistics at various steps during processing. 


\begin{tabular}{|l|l|l|l|}
\hline \multicolumn{2}{|l|}{ Table 2. Assembly statistics at various steps during processing } & \\
\hline Contig Grouping & $\begin{array}{l}\text { No. of } \\
\text { contigs }\end{array}$ & N50* & $\begin{array}{l}\text { Total sequence } \\
\text { (bp) }\end{array}$ \\
\hline Megahit assemblies 200-499bp & $24,999,285$ & n.d. & $9,293,098,676$ \\
\hline Megahit assemblies 500-1,999bp & $16,103,221$ & n.d. & $13,382,057,993$ \\
\hline Megahit assemblies $\geq 2 \mathrm{~kb}$ & $1,517,360$ & 4,658 & $6,691,877,664$ \\
\hline Megahit assemblies $\geq 2 \mathrm{~kb}$ (post-CD-HIT-EST) & $1,126,975$ & 4,520 & $4,894,479,496$ \\
\hline Minimus2 contigs & 158,414 & 15,394 & $1,727,079,865$ \\
\hline $\begin{array}{l}\text { Minimus2 + unincorporated Megahit contigs } \\
\geq 2 \mathrm{~kb} \text { (data-rich-contigs) }\end{array}$ & 660,937 & 5,466 & $3,612,405,904$ \\
\hline $\begin{array}{l}\text { Minimus2 + unincorporated Megahit contigs } \\
\geq 7.5 \mathrm{~kb} \text { (binned-contigs) }\end{array}$ & 95,506 & 20,556 & $1,725,063,313$ \\
\hline
\end{tabular}

$1 * \mathrm{~N} 50$ - length of DNA sequence above which $50 \%$ of the total is contained 